LPTENS-93/38

hep-th/9310116

October 93

\title{
INTRODUCTION TO DIFFERENTIAL W-GEOMETRY
}

\author{
Jean-Loup GERVAIS \\ Laboratoire de Physique Théorique de l'École Normale Supérieure円, \\ 24 rue Lhomond, 75231 Paris CEDEX 05, France.
}

\begin{abstract}
Ideas recently put forward by Y. Matsuo and the author are summarized on the example of the simplest $\left(W_{3}\right)$ generalization of two-dimensional gravity. These notes are based on lectures given at the workshop "Strings, Conformal Models and Topological Field Theories", Cargese 12-21 May 1993; and the meeting "String 93", Berkeley, 24-29 May 1993.
\end{abstract}

\footnotetext{
${ }^{1}$ Unité Propre du Centre National de la Recherche Scientifique, associée à l'École Normale Supérieure et à l'Université de Paris-Sud.
} 


\section{Introduction}

In many ways, W-algebras are natural generalizations of the Virasoro algebra. They were first introduced as consistent operator-algebras involving operators of spins higher than two [1]. Moreover, the Virasoro algebra is intrinsically related with the Liouville theory which is the Toda theory associated with the Lie algebra $A_{1}$, and this relationship extends to $\mathrm{W}$-algebras which are in correspondence with the family of conformal Toda systems associated with arbitrary simple Lie algebras[2]. Another point is that the deep connection between Virasoro algebra and KdV hierarchy has a natural extention]3] to $\mathrm{W}$-algebras and higher $\mathrm{KdV}(\mathrm{KP})$ hierachies[4]. On the other hand, W symmetries exhibit strikingly novel features. First, they are basically non-linear algebras. Since the transformation laws of primary fields contain higher derivatives, product of primaries are not primaries at the classical level. Naive tensor-products of commuting representations do not form representations. A related novel feature is that $\mathrm{W}$-algebras generalize the diffeomorphisms of the circle by including derivatives of degree higher than one. Going beyond linear approximation (tangent space ) is a highly non-trivial step. Taking higher order derivatives changes the shape of the world-sheet in the target-space, thus W-geometry should be related to the extrinsic geometry of the embedding. Finally, Virasoro algebras are notoriously related to Riemann surfaces. The $\mathrm{W}$-generalization of the latter notion is a fascinating problem.

In a series of recent papers, we have developed a geometrical framework for the class of Conformal field Theory CFT mentioned above, where these features emerge from the standard Riemannian geometry of particular manifolds which we called $W$ surfaces [5]-[7]. These references cover quite a lot of material, and the present lecture will go in the opposite way. Leaving the description of the general scheme to refs [5]-[7], we shall, instead, illustrate the ideas by two explicit examples: the 2D gravity case (section 2), and its simplest generalization to the $W_{3}$ gravity (section 3 ). In both cases, we discuss the two current approaches, namely, the conformal one where $\mathrm{W}$-gravity is identified with a conformal Toda (or Liouville theory for 2D gravity) theory, and the light-cone approach.

Before starting, however, let us recall how our general scheme goes. One basic point is to make use of the fact that one deals with integrable models, but geometrically there are two aspects. The first uses extrinsic geometry. In 
ref. [6], we showed that the $A_{n}$-W-geometry corresponds to the embedding of holomorphic two-dimensional surfaces in the complex projective space $C P^{n}$. These (W) surfaces are specified by embedding equations of the form $X^{A}=$ $f^{A}(z), \bar{X}^{A}=\bar{f}^{\bar{A}}(\bar{z})$, where $z$, and $\bar{z}$ are the two surface-parameters. The fact that they are functions of a single variable is equivalent to the Toda field-equations, so that this describes $\mathrm{W}$ gravity in the conformal gauge. These functions have a natural extension to $C P^{n}$ using the higher variables $z^{k}, \bar{z}^{k}$ of the Toda hierarchy of integrable flows, and this provides a local parametrization of $C P^{n}$. The original variables $z$ and $\bar{z}$ are identified with $z^{1}, \bar{z}^{1}$, respectively. For the embedding functions the extension is such that they become functions of half of the variables noted $f^{A}([z])=f^{A}\left(z^{0}, \cdots z^{n}\right)$, and $\bar{f}^{\bar{A}}([\bar{z}])=\bar{f}^{\bar{A}}\left(\bar{z}^{0}, \cdots \bar{z}^{n}\right)$ such that

$$
\frac{\partial f^{A}([z])}{\partial z^{k}}=\frac{\partial^{k} f^{A}([z])}{(\partial z)^{k}}, \quad \frac{\partial \bar{f}^{\bar{A}}([\bar{z}])}{\partial \bar{z}^{k}}=\frac{\partial^{k} \bar{f}^{\bar{A}}([\bar{z}])}{(\partial \bar{z})^{k}}
$$

One main virtue of the coordinates $z^{k}, \bar{z}^{k}$ is that, due to the last equations, higher derivaties in $z$ and $\bar{z}$ are changed to first-order ones, and this is how our geometrical scheme gets rid of the troublesome higher derivatives of the usual approachs.

The second aspect[5] [6] only makes use of intrinsic geometries, but introduces a family of associated surfaces in the standard Grassmannians associated with $C P^{n}$. This is useful to discuss global aspects by using the fact developed in ref. [6], that $\mathrm{W}$ surfaces are instantons of non-linear $\sigma$ models. We shall not dwell into this aspect in these lectures.

So far this is only for the conformal gauge. Concerning the light-cone approach, our recent insight[0] is that, for any Kähler manifold, there exist changes of coordinates such that metric tensors of the light-cone type come out. This allows us to relate conformal and light-cone descriptions by diffeomorphisms.

Finally, let us stress that we shall remain entirely at the level of classical field theory, thus describing only the $C \rightarrow \infty$ limit of the problem. The quantum approach to Toda theories is making steady progress [9]-[15], [18][20] but its connection with the present geometrical scheme remains as a fascinating problem for the future. 


\section{Two-dimensional Gravity}

In this part we discuss the case of two-dimensional gravity in some details, using methods that will be later on generalized to $\mathrm{W}$ gravity. With general world-sheet parameters $\xi$, the Weyl anomaly takes the form

$$
S=\frac{1}{\gamma} \int d_{2} \xi\left(\frac{1}{2} \mathcal{R} \frac{1}{\Delta} \mathcal{R}+\mu \sqrt{-\mathcal{G}}\right)
$$

In this expression, $\Delta$ is the Laplacian with the $2 \mathrm{D}$-gravity metric $\mathcal{G}_{\alpha, \beta}, \gamma$ is the coupling constant, $\mathcal{R}$ the scalar curvature, and $\mu$ the cosmological constant.

\subsection{Conformal gauge}

First, choose conformal coordinates $z$ and $\bar{z}$, so that the arc length takes the form 2

$$
d s^{2}=2 e^{2 \Phi} d z d \bar{z},
$$

the Weyl anomaly becomes the Liouville action

$$
S=\frac{1}{\gamma} \int d_{2} z\left(-\frac{1}{2} \partial \Phi \bar{\partial} \Phi+\mu e^{2 \Phi}\right)
$$

In general $\partial$ and $\bar{\partial}$ denote $\partial / \partial z$, and $\partial / \partial \bar{z}$ respectively. At the level of the present discussion we may always set $\mu=1$ by a shift of $\phi$, and we shall do so from now on. The general solution of the Liouville equation is given by the holomorphic decomposition

$$
e^{-\Phi}=\sum_{j=1}^{2} \chi_{j}(z) \bar{\chi}_{j}(\bar{z}) .
$$

The functions $\chi_{j}(z)$, and $\bar{\chi}_{j}(\bar{z})$ are pairs of solutions of the differential equations

$$
-\frac{d^{2} \chi_{j}(z)}{(d z)^{2}}+T(z) \chi_{j}(z)=0, \quad-\frac{d^{2} \bar{\chi}_{j}(\bar{z})}{(d \bar{z})^{2}}+\bar{T}(\bar{z}) \bar{\chi}_{j}(\bar{z})=0
$$

\footnotetext{
${ }^{2}$ This may not be possible globally, but we shall only consider the local aspects
} 
where $T$ and $\bar{T}$ are the two non-vanishing components of the stress-energy tensor. They are normalized so that

$$
\chi_{1}(z) \frac{d \chi_{2}(z)}{d z}-\chi_{2}(z) \frac{d \chi_{1}(z)}{d z}=\bar{\chi}_{1}(\bar{z}) \frac{d \bar{\chi}_{2}(\bar{z})}{d \bar{z}}-\chi_{2}(\bar{z}) \frac{d \chi_{1}(\bar{z})}{d \bar{z}}=1 .
$$

At this point, and in the following, we need a simple mathematical lemma concerning differential equations, which we state once for all next.

Lemma 1 Automatic differential equation.

Consider $N$ functions $f^{A}$ of a single variable $x$, whose Wronskian does not vanish. They satisfy a differential equation of the form

$$
f^{(N) A}(x)=\sum_{\ell=1}^{N} \kappa_{\ell}(x) f^{(N-\ell) A}(x) .
$$

Proof: Recall the definition of the Wronskian:

$$
\operatorname{Wr}\left(f^{1}, \cdots, f^{N}\right) \equiv\left|\begin{array}{ccc}
f^{1} & \cdots & f^{N} \\
f^{(1) 1} & \cdots & f^{(1) N} \\
\vdots & \cdots & \vdots \\
f^{(N-1) 1} & \cdots & f^{(N-1) N}
\end{array}\right| .
$$

In this formula, and hereafter, upper indices in between parentheses denote the order of derivatives. Clearly, any of the functions $f^{A}$ may be trivially written as a linear combination of the type $\sum_{B} c_{B}^{A} f^{B}$. It is then clear that

$$
\left|\begin{array}{cccc}
f^{1} & \cdots & f^{N} & f \\
f^{(1) 1} & \cdots & f^{(1) N} & f^{(1)} \\
\vdots & \cdots & \vdots & \\
f^{(N) 1} & \cdots & f^{(N) N} & f^{(N)}
\end{array}\right|=0
$$

Expanding this determinant with respect to the last column immediately gives Eq.2.7. Q.E.D. Moreover, one immediately sees that

$$
\kappa_{1}=\frac{d \ln \left[\operatorname{Wr}\left(f^{1}, \cdots, f^{n}\right)\right]}{d x}
$$

Returning to our main line, we see that, in the differential equations Eq.2.5 the first order term vanishes, so that the Wronskians $\operatorname{Wr}(\chi)$ and $\operatorname{Wr}(\bar{\chi})$ 
are constant, and may be chosen equal to one (see Eq.2.6). For our geometrical description this is not appropriate, however. The basic reason is as follows. Under conformal transformations $\delta z=\epsilon(z)$, the $\chi_{j}$ fields transform as primary fields of weight $-1 / 2$ (that is such that $\chi_{j}(z)(d z)^{-1 / 2}$ is invariant). This is consistent, since it follows that the Wronskian $\operatorname{Wr}(\chi)$ transforms with weight zero, so that condition Eq.2.6 is conformally invariant. In our geometrical description, these functions will become geometrical objects whose form should not change under conformal transformation, so that they should transform with weight zero. We may change the conformal weights by using a projective description. For this we define

$$
f^{A}=\sqrt{w(z)} \chi_{A+1}, \quad \bar{f}^{A}=\sqrt{\bar{w}(\bar{z})} \bar{\chi}_{A+1}, \quad A=0,1,
$$

so that

$$
\operatorname{Wr}\left(f^{0}, f^{1}\right)=w(z), \quad \operatorname{Wr}\left(\bar{f}^{0}, \bar{f}^{1}\right)=\bar{w}(z)
$$

where $w$ and $\bar{w}$ are arbitary functions of a single variable. Now the f's satisfy a more general differential equation of the type Eq.2.7, where $\kappa_{1} \neq 0$. Substituting into Eq.2.4, we derive the arc length

$$
d s^{2}=d z d \bar{z} \frac{\operatorname{Wr}\left(f^{0}, f^{1}\right) \operatorname{Wr}\left(\bar{f}^{0}, \bar{f}^{1}\right)}{\left(f^{0} \bar{f}^{0}+f^{1} \bar{f}^{1}\right)^{2}},
$$

and, re-arranging the terms, we may write

$$
\begin{gathered}
d s^{2}=d z d \bar{z}\left(\frac{-\left(f^{(1) 0} \bar{f}^{(1) 0}+f^{(1) 1} \bar{f}^{(1) 1}\right)\left(f^{0} \bar{f}^{0}+f^{1} \bar{f}^{1}\right)}{\left(f^{0} \bar{f}^{0}+f^{1} \bar{f}^{1}\right)^{2}}+\right. \\
\left.\frac{\left(f^{(1) 0} \bar{f}^{0}+f^{(1) 1} \bar{f}^{1}\right)\left(f^{0} \bar{f}^{(1) 0}+f^{1} \bar{f}^{(1) 1}\right)}{\left(f^{0} \bar{f}^{0}+f^{1} \bar{f}^{1}\right)^{2}}\right) .
\end{gathered}
$$

As is well-known $\operatorname{Wr}\left(\rho f^{0}, \rho f^{1}\right)=\rho^{2} \operatorname{Wr}\left(f^{0}, f^{1}\right)$, for arbitrary function $\rho$, so that Eq.2.12 is invariant under $f^{A} \rightarrow \rho f^{A}$, and $\bar{f}^{A} \rightarrow \bar{\rho} \bar{f}^{A}$. We arrive at a projective structure, following our general scheme[6] where $A_{n}$-W geometries are described in $C P^{n}$ (we will have $n=1$ in the present case). Let us recall some useful definitions at this point.

\footnotetext{
${ }^{3}$ In the conformal gauge $\chi$ and $\bar{\chi}$ are on the same footing. When we discuss properties of chiral component we some times talk about the $\chi$ fields as an example. Clearly, the $\bar{\chi}$ fields are analogous.

${ }^{4}$ this is obviously true by construction.
} 


\section{Definition 1 Kähler manifolds}

A Kähler manifold of real dimension $2 n$ is complex manifold with a special class of coordinates $X^{A}, X^{\bar{A}}, 1 \leq A, \bar{A} \leq n$, such that the only components of the metric are $G_{A \bar{B}}=G_{\bar{B} A}$, and

$$
G_{A \bar{B}}=\partial_{A} \partial_{\bar{B}} K,
$$

where $K$ is the Kähler potential.

In general we denote the differential operators $\partial / \partial X^{A}$, and $\partial / \partial X^{\bar{B}}$ by $\partial_{A}$, and $\partial_{\bar{B}}$.

Definition 2 Complex projective space $C P^{n}$

The complex projective space $C P^{n}$ is defined from the trivial complex space $C^{n+1}$ with coordinates $X^{A}, \bar{X}^{\bar{A}}, A, \bar{A}=0, \cdots n$, by identifying any two points $X, \bar{X}$, and $Y, \bar{Y}$ related by the scale transformation

$$
X^{A}=Y^{A} \rho, \quad \text { and } \quad \bar{X}^{\bar{A}}=\bar{Y}^{\bar{A}} \bar{\rho} .
$$

This is a simplest non-trivial example of a Kähler manifold. The metric on this space is given by the Fubini-Study equation

$$
G_{A \bar{A}}=\left(\delta_{A \bar{A}}\left(\sum_{B=0}^{n} X^{B} \bar{X}^{B}\right)-X^{\bar{A}} \bar{X}^{A}\right) /\left(\sum_{B=0}^{n} X^{B} \bar{X}^{B}\right)^{2},
$$

whose Kähler potential is given by

$$
K=\ln \sum_{A=0}^{n} X^{A} \bar{X}^{\bar{A}} .
$$

Eq.2.16 is invariant under the scale transformation Eq.2.15. Using this freedom, it is customary to parametrize $C P^{n}$, by letting one component (say, $X^{0}$ ) equal 1.

Closing the parenthesis, we return to Eq.2.13. Consider the space $C P^{1}$. The equations

$$
X^{A}=e^{\zeta} f^{A}(z), \quad \bar{X}^{A}=e^{\bar{\zeta}} \bar{f}^{A}(\bar{z}), \quad A=0,1,
$$

define a holomorphic change of coordinates in $C^{2}$ from $X^{A}$ to $\zeta, z$. It is easy to see that Eq.2.13 is equivalent to

$$
G_{z \bar{z}} \equiv e^{2 \Phi}=\left.f^{(1) A} \bar{f}^{(1) \bar{B}} G_{A \bar{B}}\right|_{X^{A}=f^{A}, \bar{X}^{\bar{A}}=\bar{f}^{\bar{A}}}
$$


By construction, Eq.2.13 is invariant under the rescaling $f \rightarrow \rho(z) f, \bar{f} \rightarrow$ $\bar{\rho}(\bar{z}) \bar{f}$. Thus we may let $\zeta=\bar{\zeta}=0$, and the geometrical meaning of Eqs.2.18, Eq.2.19 is that $z \bar{z}$ are parameters of $C P^{1}$, such that the Liouville exponential $G_{z, \bar{z}}$ is equivalent to the metric tensor of Fubini-Study. Thus any solution of Liouville equation defines a local holomorphic parametrization of $C P^{1}$. Note that, since the change of coordinate is holomorphic, the Kähler condition is preserved. Indeed, one has

$$
G_{z \bar{z}}=\partial \bar{\partial} \mathcal{K}, \quad \mathcal{K}=\ln \left(f^{0} \bar{f}^{0}+f^{1} \bar{f}^{1}\right) .
$$

\subsection{Light-cone gauge}

\subsubsection{The Weyl anomaly}

First, rederive the ideas of ref. [8] is the present context. At the level of Eq.2.1, one changes coordinates from $z \bar{z}$ to $u \bar{u}$, such that

$$
e^{2 \Phi} d z d \bar{z}=d u d \bar{u}+h d u^{2}
$$

The Weyl anomaly Eq.2.1 becomes

$$
S=\frac{1}{2 \gamma} \int d_{2} u\left(\left(\bar{D}^{2} h\right) \frac{1}{D \bar{D}-\bar{D} h \bar{D}}\left(\bar{D}^{2} h\right)+2\right),
$$

where $D$ and $\bar{D}$ stand for $\partial / \partial u$, and $\partial / \partial \bar{u}$, respectively. Here the second term is a constant and can be forgotten. Using the obvious relation

$$
\frac{1}{D \bar{D}-\bar{D} h \bar{D}}=\frac{1}{D-h \bar{D}} \frac{1}{\bar{D}}
$$

we may write after partially integrating

$$
S=\frac{1}{\pi} \int d_{2} u h \mathcal{T}, \quad \mathcal{T} \equiv \frac{\pi}{2 \gamma} \bar{D}^{2} \frac{1}{D-h \bar{D}} \bar{D} h
$$

Starting from this expression, we compute

$$
(D-h \bar{D}-2(\bar{D} h)) \mathcal{T}=\frac{\pi}{2 \gamma} \bar{D}^{2} \frac{D-h \bar{D}}{D-h \bar{D}} \bar{D} h
$$

so that we get

$$
(D-h \bar{D}-2(\bar{D} h)) \mathcal{T}=\frac{\pi}{2 \gamma} \bar{D}^{3} h
$$


Thus the deformed conservation law are satisfied. Minimizing $S$, under the form Eq.2.23, with respect to $h$, one finds

$$
0=\frac{\pi}{2 \gamma} \bar{D}^{2} \frac{1}{D-h \bar{D}} \bar{D} h=\mathcal{T}
$$

so that Polyakov's anomaly equation comes out:

$$
\bar{D}^{3} h=0
$$

\subsubsection{Liouville dynamics}

Next, following ref. [7], we connect the results just recalled with the conformalgauge Liouville appproach recalled above. Since the Liouville equations also follow from minimizing $S$, they are equivalent to Polyakov's anomaly equations. This may be seen as follows. Making use of Eq.2.21, one sees that

$$
h=-\frac{\partial \bar{u}}{\partial z}, \quad e^{2 \Phi}=\frac{\partial \bar{u}}{\partial \bar{z}},
$$

The last equation is immediately solved, thanks to Eq.2.20, and we find that the change of variables form conformal to light-cone explicitly reads

$$
u=z, \quad \bar{u}=\partial K=\frac{f^{(1) 0} \bar{f}^{0}+f^{(1) 1} \bar{f}^{1}}{f^{0} \bar{f}^{0}+f^{1} \bar{f}^{1}}
$$

Computing $h$ in terms of $f^{1}$, and $\bar{f}^{1}$ one finds.

$$
h=-\frac{f^{(2) 0} \bar{f}^{0}+f^{(2) 1} \bar{f}^{1}}{f^{0} \bar{f}^{0}+f^{1} \bar{f}^{1}}+\left[\frac{f^{(1) 0} \bar{f}^{0}+f^{(1) 1} \bar{f}^{1}}{f^{0} \bar{f}^{0}+f^{1} \bar{f}^{1}}\right]^{2}
$$

The first term is retransformed by using the lemma which gives $f^{(2) A}=$ $\kappa_{1} f^{(1) A}+\kappa_{0} f^{A}$. One gets

$$
h=-\kappa_{0}-\kappa_{1} \bar{u}+\bar{u}^{2}
$$

For fixed $z=u$, it is quadratic in $\bar{u}$, so that Eq.2.25 is indeed verified. In terms of the $\operatorname{sl}(2, R)$ light-cone currents we have $J^{+}=1, J^{0}=\kappa_{1} / 2$, and $J^{-}=-\kappa_{0}$. 


\section{$3 \quad W_{3}$ gravity}

Unfortunately no formula similar to the Weyl-anomaly term Eq.2.1 is known at present. Our starting point will be the generalization of the Liouville equation known as the $A_{2}$ Toda equation, where the $W_{3}$ algebra is realized by Poisson bracket, and which should thus describe $W_{3}$ gravity in the conformal gauge.

\subsection{Conformal gauge}

\subsection{1 $W$ surface}

In the $A_{2}$ Toda theory there are two fields $\phi_{1}, \phi_{2}$. The field equations read

$$
\partial \bar{\partial} \phi_{1}=-e^{2 \phi_{1}-\phi_{2}}, \quad \partial \bar{\partial} \phi_{2}=-e^{2 \phi_{2}-\phi_{1}}
$$

with solution

$$
e^{-\phi_{1}}=\sum_{j=1}^{3} \chi_{j}(z) \bar{\chi}_{j}(\bar{z}), \quad e^{-\phi_{2}}=\sum_{i<j=1}^{3} \chi_{i j}(z) \bar{\chi}_{i j}(\bar{z})
$$

There are now three pairs of arbitary functions of a single variable $\chi_{j}, \bar{\chi}_{j}$, the functions $\chi_{i j}$, and $\bar{\chi}_{i j}$ are defined by

$$
\chi_{i j}=\operatorname{Wr}\left(\chi_{i}, \chi_{j}\right), \quad \bar{\chi}_{i j}=\operatorname{Wr}\left(\bar{\chi}_{i}, \bar{\chi}_{j}\right) .
$$

The Toda equations 3.1 are satisfied if

$$
\operatorname{Wr}\left(\chi_{1}, \chi_{2}, \chi_{2}\right)=1, \quad \operatorname{Wr}\left(\bar{\chi}_{1}, \bar{\chi}_{2}, \bar{\chi}_{2}\right)=1,
$$

One sees that the generalization of the Liouville case is very direct. The lemma, combined with the last Wronskian condition shows that the $\chi_{j}$ satisfy a differential equation of the form

$$
\chi_{j}^{(3)}=T \chi_{j}^{(1)}+W \chi_{j}
$$

The potentials are the generators of the $W$ transformations: $T$ is the stressenergy tensor, and $W$ the $W_{3}$ charge. Again we relax the condition 3.4 by letting

$$
f^{A}=(w(z))^{1 / 3} \chi_{A+1}, \quad \bar{f}^{A}=(\bar{w}(\bar{z}))^{1 / 3} \bar{\chi}_{A+1}, \quad A=0,1,2
$$


so that

$$
\begin{gathered}
\operatorname{Wr}\left(f^{0}, f^{1}, f^{2}\right)=w(z), \quad \operatorname{Wr}\left(\bar{f}^{0}, \bar{f}^{1}, \bar{f}^{2}\right)=\bar{w}(z) \\
e^{-\phi_{1}}=\frac{\sum_{A=0}^{2} f^{A}(z) \bar{f}^{A}(\bar{z})}{\left[\operatorname{Wr}\left(f^{0}, f^{1}, f^{2}\right) \operatorname{Wr}\left(\bar{f}^{0}, \bar{f}^{1}, \bar{f}^{2}\right)\right]^{1 / 3}} \\
e^{-\phi_{2}}=\frac{\sum_{A<B=0}^{2} \operatorname{Wr}\left(f^{A}, f^{B}\right) \operatorname{Wr}\left(\bar{f}^{A}, \bar{f}^{B}\right)}{\left[\operatorname{Wr}\left(f^{0}, f^{1}, f^{2}\right) \operatorname{Wr}\left(\bar{f}^{0}, \bar{f}^{1}, \bar{f}^{2}\right)\right]^{2 / 3}}
\end{gathered}
$$

Our task is now to give a meaning for these formulae in Riemannian geometry. There are two new features as compared with the Liouville case. First we now have three pairs of functions, but we still only have two variables $z$ and $\bar{z}$. Second, derivatives of second degree appear so that one does not have a direct tangent-space interpretation. These two problems are solved simultaneously according to our general scheme. First, we introduce $C P^{2}$ as target space where the equations

$$
X^{A}=f^{A}(z), \quad \bar{X}^{A}=\bar{f}^{A}(\bar{z}), \quad A=0,1,2,
$$

define a holomorphic $(W)$ surface. Second, we will also consider the extrinsic geometry of these surfaces where higher derivatives naturally appear when one defines normals. It is convenient to define

$$
\widetilde{G}_{a \bar{b}}=\left.\sum_{A, \bar{B}} f^{(a) A}(z) \bar{f}^{(\bar{b}) \bar{B}}(\bar{z}) G_{A \bar{B}}\right|_{X^{A}=f^{A}, \bar{X}^{\bar{A}}=\bar{f}^{\bar{A}}}=\widetilde{G}_{\bar{b} a}
$$

Consider first the intrinsic geometry. It is straightforward to see that, according to Eq.3.8, the arc-length on the surface is given by

$$
d s^{2} \equiv \widetilde{G}_{11} d z d \bar{z}=e^{2 \phi_{1}-\phi_{2}} d z d \bar{z}
$$

so that this particular exponential of the Toda fields gives the induced metric on the $W$ surface. The complete geometrical interpretation may be obtained following the usual method of differential geometry, namely by considering normals to the surface. There are two of them, noted $e_{1}$, and $e_{\overline{1}}$. Their components may be written compactly by the generalized Frenet-Serret formulae

$$
e_{1}^{A}=\frac{1}{\sqrt{\widetilde{G}_{1 \overline{1}} \Delta_{2}}}\left|\begin{array}{cc}
\widetilde{G}_{1 \overline{1}} & \widetilde{G}_{2 \overline{1}} \\
f^{(1) A} & f^{(2) A}
\end{array}\right|, \quad e_{1}^{\bar{A}}=0
$$




$$
e_{\overline{1}}^{A}=0, \quad e_{\overline{1}}^{\bar{A}}=\frac{1}{\sqrt{\widetilde{G}_{1 \overline{1}} \Delta_{2}}}\left|\begin{array}{cc}
\widetilde{G}_{1 \overline{1}} & \widetilde{G}_{1 \overline{2}} \\
\bar{f}^{(1) \bar{A}} & \bar{f}^{(2) \bar{A}}
\end{array}\right|
$$

where

$$
\Delta_{2} \equiv\left|\begin{array}{cc}
\widetilde{G}_{1 \overline{1}} & \widetilde{G}_{1 \overline{2}} \\
\widetilde{G}_{2 \overline{1}} & \widetilde{G}_{2 \overline{2}}
\end{array}\right|
$$

It is easy to see that, if we denote by $(\mathbf{X}, \mathbf{Y})$ the inner product $G_{A \bar{B}}\left(X^{A} Y^{\bar{B}}+\right.$ $\left.Y^{A} X^{\bar{B}}\right)$ in $C P^{2}$, we have $\left(e_{1}, f^{(1)}\right)=0,\left(e_{1}, \bar{f}^{(1)}\right)=0,\left(\bar{e}_{1}, f^{(1)}\right)=0,\left(\bar{e}_{1}, \bar{f}^{(1)}\right)=$ $0,\left(e_{1}, e_{1}\right)=\left(e_{\overline{1}}, e_{\overline{1}}\right)=0,\left(e_{1}, e_{\overline{1}}\right)=1$. So that the last equations define orthonormalized normals. With the present notations, the second fundamental form $Q$ is obtained by writing the equivalent expressions

$$
f^{(2) A}-f^{(1) A} \frac{\widetilde{G}_{2 \overline{1}}}{\widetilde{G}_{1 \overline{1}}}=e_{1}^{A} Q, \quad \bar{f}^{(2) \bar{A}}-\bar{f}^{(1) \bar{A}} \frac{\widetilde{G}_{2 \overline{1}}}{\widetilde{G}_{1 \overline{1}}}=e_{\overline{1}}^{\bar{A}} Q
$$

with $Q=\sqrt{\Delta_{2} / \widetilde{G}_{1 \overline{1}}}$. By explicit computation one finds that $\Delta_{2}=\exp \left(3 \phi_{1}\right)$, so that

$$
Q=e^{\left(\phi_{1}+\phi_{2}\right) / 2}=\sqrt{\frac{\operatorname{Wr}\left(f^{0}, f^{1}, f^{2}\right) \operatorname{Wr}\left(\bar{f}^{0}, \bar{f}^{1}, \bar{f}^{2}\right)}{\sum_{A=0}^{2} f^{A}(z) \bar{f}^{A}(\bar{z}) \sum_{C<B=0}^{2} \operatorname{Wr}\left(f^{C}, f^{B}\right) \operatorname{Wr}\left(\bar{f}^{C}, \bar{f}^{B}\right)}} .
$$

\subsubsection{Associated holomorphic change of coordinates}

Although the geometrical meaning of the Toda fields is now clear, the description is not covariant under change of parametrization of $C P^{2}$, since it involves second derivatives. This problem is solved by using the fact that the Toda field equations are a subsystem of the so-called Toda hierarchy of integrable flows that makes use of 6 real parameters. These parameters provide a parametrization of $C^{3}$ where the formulae we summarized may be re-expressed in terms of first order derivatives only, so that their covariance properties become clear. This part is best handled by means of the fermionic method and Hirota equations [4] [5] [6]. We shall nevertheless remain at the same elementary simple-minded level for illustration. In terms of $f$ and $\bar{f}$, the Toda hierarchy equations become extremely simple (see Eq.1.1). The

\footnotetext{
5 this is done more powerfully using the fermionic method as explained in ref. 6 ]
} 
variables $\operatorname{are} \oint^{\text {(0 }} z^{0}, z^{1} \equiv z, z^{2}$ denoted collectively as $[z]$; and $\bar{z}^{0}, \bar{z}^{1} \equiv \bar{z}, \bar{z}^{2}$ denoted collectively as $[\bar{z}]$. The functions $f^{A}(z)$ and $\bar{f}^{\bar{A}}(\bar{z})$ are extended as holomorphic or antiholomorphic functions $f^{A}([z])$ and $\bar{f}^{\bar{A}}([\bar{z}])$ solutions of the equations

$$
\begin{array}{lll}
\partial_{0} f^{A}([z])=f^{A}([z]), & \partial_{2} f^{A}([z])=\left(\partial_{1}\right)^{2} f^{A}([z]) \\
\bar{\partial}_{0} \bar{f}^{\bar{A}}([\bar{z}])=\bar{f}^{\bar{A}}([\bar{z}]), & \bar{\partial}_{2} \bar{f}^{\bar{A}}([\bar{z}])=\left(\bar{\partial}_{1}\right)^{2} \bar{f}^{\bar{A}}([z])
\end{array}
$$

with the initial conditions

$$
f^{A}([z])=f^{A}(z), \quad \bar{f}^{\bar{A}}(\bar{z})=\bar{f}^{\bar{A}}([\bar{z}]), \quad \text { for } z^{0}=z^{2}=\bar{z}^{0}=\bar{z}^{2}=0
$$

Of course the differential equations in $z^{0}$ and $\bar{z}^{0}$ are trivial and give simple factors $\exp \left(z^{0}\right)$ or $\exp \left(\bar{z}^{0}\right)$. Since we work homogeneously we may forget these variables completely and keep $z^{0}$ and $\bar{z}^{0}$ equal to zero. The point of the differential equations in $z^{2}$ is to turn second derivatives in $z$ into a first order derivatives. Thus the matrix of inner product of derivatives Eq.3.10 is extended to $C P^{2}$ as

$$
G([z],[\bar{z}])_{a \bar{b}}=\left.\sum_{A, \bar{B}} \partial_{a} f^{A}([z]) \partial_{\bar{b}} \bar{f}^{\bar{B}}([\bar{z}]) G_{A \bar{B}}\right|_{X^{A}=f^{A}([z]), \bar{X}^{\bar{A}}=\bar{f}^{\bar{A}}([\bar{z}])}
$$

where now only first order derivatives appear. As a matter of fact, this last equation is but the Riemannian metric obtained by making the holomorphic change of variables $X^{A}=f^{A}\left(z, z^{2}\right), \bar{X}^{\bar{A}}=\bar{f}^{\bar{A}}\left(\bar{z}, \bar{z}^{2}\right)$. Thus again, we associate, with any solution of the Toda equation, a conformal parametrization of the complex projective space. Note again that, since the change is conformal, the Kähler condition is preserved. The metric $G([z],[\bar{z}])_{a \bar{b}}$ may be written as

$$
G([z],[\bar{z}])_{a \bar{b}}=\partial_{a} \partial_{\bar{b}} \ln \left[\sum_{A} f^{A}([z]) \bar{f}^{A}([\bar{z}])\right]
$$

Our next topic is the $\mathrm{W}$ transformations of $f$. We shall use the parametrization such that $X^{0}=\bar{X}^{0}=1$, so that $f^{0}([z])=\bar{f}^{0}([\bar{z}])=1$. Due to these

\footnotetext{
${ }^{6}$ Upper indices are always covariant indices. When we want to indicate powers such as $z$ square, we put parentheses, and write $(z)^{2}$.
} 
conditions, and according to the lemma, the functions $f^{A}(z)$ are solution of a differential equation of the form

$$
f^{(3) A}(z)=\lambda(z) f^{(2) A}(z)+\mu(z) f^{(1) A}(z)
$$

there is no term without derivative since $f^{0}=1$ is a solution. The most general infinitesimal transformation that leaves the condition $f^{0}=1$ invariant is

$$
\delta f^{A}(z)=\left(\alpha(z) \partial+\beta(z) \partial^{2}\right) f^{A}(z)
$$

Higher derivatives may be re-expressed in terms of the first two by means of Eq.3.21. Consider, now the extension to $C P^{2}$. To simplify the notation we will let $t=z^{2}$, so that, according to Eqs.3.17,

$$
\partial_{t} f^{A}(z, t)=(\partial)^{2} f^{A}(z, t) .
$$

This is used in practice as follows. Consider

$$
\delta_{n} f(z)=\epsilon(z)(\partial)^{n} f(z)
$$

Clearly one has

$$
f^{A}(z, t)=e^{t(\partial)^{2}} f^{A}(z, 0)
$$

One writes

$$
\delta_{n} f(z, t)=e^{t(\partial)^{2}} \delta f(z)=\epsilon(z+2 t \partial) e^{t(\partial)^{2}} \partial^{n} f(z)=\epsilon(z+2 t \partial)(\partial)^{n} f(z, t)
$$

where $\epsilon(z+2 t \partial)$ is defined by

$$
\epsilon(z+2 t \partial)=\sum_{r} \frac{\epsilon_{r}}{r !}(z+2 t \partial)^{r}, \quad \epsilon_{r}=\left.(\partial)^{r} \epsilon(z)\right|_{z=0}
$$

In general, one sees that an arbitrary $W$ transformation leads to

$$
\delta f(z, t)=\alpha(z+2 t \partial) \partial f(z, t)+\beta(z+2 t \partial) \partial_{t} f(z, t)
$$

Eq.3.21 is straightforwardly extended to $C P^{2}$ where it takes the form

$$
\partial_{z} \partial_{t} f^{A}([z])=L([z]) \partial_{t} f^{A}([z])+M([z]) \partial_{z} f^{A}([z]) .
$$

Using this relation, and Eq.3.23, we may re-express all higher derivetives in Eq.3.26 in terms of the first-order ones, obtaining a transformation of form

$$
\delta f(z, t)=A(z, t) \partial f(z, t)+B(z, t) \partial_{t} f(z, t)
$$

which correspond to the change of parametrization

$$
\delta z=A(z, t), \quad \delta t=B(z, t), \text { with } \delta f(z, t)=f(z+\delta z, t+\delta t)-f(z, t) .
$$

Thus W transformations are extended as particular diffeomorphisms of $C P^{2}$. 


\subsection{Light-cone description}

Finally, we connect the above discussion with the light-cone approach to $W_{3}$ gravity, which so far was developed independently of the conformal gauge [16], [17]. The basic point, displayed in ref.[0], is that, for any Kähler manifold, there exist parametrizations such that the metric takes a blockform identical to the light-cone metric Eq.2.21 introduced by Polyakov for two-dimensional gravity.

\subsubsection{Light-cone parametrization for Kähler manifolds}

Consider an arbitrary Kähler manifold, introduced by Definition 1 . There exists another class of prefered coordinates denoted $U^{A}$, and $U^{\bar{A}}$, such that the new metric tensor denoted by the letter $H$ takes a form which is the generalization of the right-hand side of Eq.2.21. The change of coordinates is of the form

$$
U^{\bar{A}}=U^{\bar{A}}\left(X^{1}, \cdots, X^{n} ; X^{\overline{1}} \cdots, X^{\bar{n}}\right), \quad U^{A}=X^{A} .
$$

The functions $U^{\bar{A}}(X ; \bar{X})$ will be determined next, in such a way that the light-cone metric takes the form

$$
\begin{gathered}
H_{\bar{A} \bar{B}}=0, \quad H_{A B}=2 h_{A B} \\
H_{A \bar{B}}=H_{\bar{B} A}=\delta_{A, \bar{B}},
\end{gathered}
$$

where $h_{A B}$ will be related to the Kähler potential. Before going on, let us remark that the determinant of $H$ is equal to -1 , and that its inverse is given by

$$
\begin{gathered}
H^{A B}=0, \quad H^{\bar{A} \bar{B}}=-2 h_{A B} \\
H^{A \bar{B}}=H^{\bar{B} A}=\delta_{A, \bar{B}} .
\end{gathered}
$$

On the contrary, no general form may be given for the determinant of $G$ or its inverse. This is a very nice point of the light-cone parametrization?. Going back to our main line, one finds, by standard computations, that conditions Eqs.3.31 are fulfilled if one has

$$
G_{A \bar{B}}=H_{A \bar{C}} \partial_{\bar{B}} U^{\bar{C}}
$$

\footnotetext{
${ }^{7}$ inverting the metric tensor is often a pain in the neck.
} 


$$
2 h_{A B}=-H_{B \bar{C}} \partial_{A} U^{\bar{C}}-H_{A \bar{C}} \partial_{B} U^{\bar{C}}
$$

These equations are easily solved by using the Kähler potential, obtaining

$$
\begin{aligned}
& U^{\bar{A}}=H^{\bar{A} C} \partial_{C} K, \\
& h_{A B}=-\partial_{A} \partial_{B} K .
\end{aligned}
$$

Thus we reach the important conclusion that, for any Kählerian manifold there is a choice of coordinates such that the metric tensor takes the blockform

$$
H=\left(\begin{array}{cc}
2 h & 1 \\
1 & 0
\end{array}\right)
$$

which is the same as the one introduced by Polyakov to describe 2D gravity in the light-cone gauge.

\subsubsection{Light-cone coordinates for $W_{3}$ gravity}

We finally apply this change of coordinates to $C P^{2}$, calling $\bar{u}^{1}$, and $\bar{u}^{2}$ the two light-cone coordinates. Combining Eq.3.21 with Eq.3.35, 3.36, one sees that one has

$$
\begin{gathered}
\bar{u}^{\ell}=\frac{\sum_{A=0}^{2} \partial_{\ell} f^{A}([z]) \bar{f}^{A}([\bar{z}])}{\sum_{A=0}^{2} f^{A}([z]) \bar{f}^{A}([\bar{z}])} \\
h_{\ell m}=-\frac{\sum_{A=0}^{2} \partial_{\ell} \partial_{m} f^{A}([z]) \bar{f}^{A}([\bar{z}])}{\sum_{A=0}^{2} f^{A}([z]) \bar{f}^{A}([\bar{z}])}+\bar{u}^{\ell} \bar{u}^{m}
\end{gathered}
$$

Making use of Eq.3.27, this gives

$$
\begin{gathered}
h_{11}=-\bar{u}^{2}+\left(\bar{u}^{1}\right)^{2}, \quad h_{12}=-L \bar{u}^{2}-M \bar{u}^{1}+\bar{u}^{1} \bar{u}^{2} \\
h_{22}=-\bar{u}^{2}\left(\partial L+L^{2}+M\right)-\bar{u}^{1}(\partial M+L M)+\left(\bar{u}^{2}\right)^{2}
\end{gathered}
$$

so that $h$ is quadratic in the $\bar{u}$ 's. On the other hand, Polyakov's equation for light-cone $W_{3}$ gravity takes the form $(\bar{D})^{3} h=0$, and $(\bar{D})^{5} A=0$. This contradiction may be resolved as follows: The $\mathrm{W}$ surface where light-cone physics takes place is not at $z^{(2)}=\bar{z}^{(2)}=0$, contrary to conformal physics. Indeed, let us assume that the light-cone W surface has equations

$$
z^{(2)}=0, \quad \bar{u}^{2}=\alpha(z)\left(\bar{u}^{1}\right)^{2}+\beta(z) \bar{u}^{1}+\gamma(z)
$$


Then, on this surface, $h_{\ell m}$ is a polynomial in $\bar{U}^{1}$ of degree $\ell+m$. Thus it seems natural to let

$$
h_{11}=h, \quad h_{22}=A
$$

obtaining

$$
(\bar{D})^{3} h=0, \quad(\bar{D})^{5} A=0, \quad \text { with } \bar{D}=\bar{D}_{1}
$$

Clearly, $\alpha, \beta$, and $\gamma$ are arbitrary. They may be precisely changed by an $\operatorname{sl}(3)$ transformation. Thus, one is led to conjecture that the geometrical origin of the $\operatorname{sl}(3)$ invariance is a change of the light-cone $\mathbf{W}$ surface so that Eq.3.39 keeps its form.

\section{Outlook}

These lecture notes left out many aspects, some of which have been developed elsewhere. In particular, quantization has led to many interesting progress. For the Liouville case, it was realized [12, 9] that there exists a quantum-group structure where the mathematical parameter of the quantum-group deformation coincides with Planck's constant. In this theory, the non-commutativity which is inherent in the quantum group - since the co-product in nonsymmetric - is brought about by the actual quantization of the system. This appearance of quantum group seems to be very natural geometrically, since one deals with a gravity theory, where the space-time metric is quantized, which seems tantamount to quantizing the $2 \mathrm{D}$ space-time itself. Thus an object like a quantum plane should appear, and quantum groups are natural transformations of such "quantum manifolds". For recent progress in this direction, see refs. [18, 19, 20]. The quantum group picture was extended to the quantum $A_{n}$ Toda theories in ref.[11]. The classical geometry setting summarized in these notes indicates that at the quantum level one should be dealing with quantum complex projective spaces. This remains to be clarified.

Classically, what we describe is also incomplete. In the light-cone connection, the ansatz Eq.3.41 remains somewhat ad hoc, and its Kac-Moody structure should be clarified. Since we use the Toda equation throughout, we stayed on the mass shell. Thus we only get Polyakov equations (Eqs.2.25, 3.42), and not the full anomaly equations that characterize light-cone $W$

gravities. Besides the particular change of gauge we have discussed, the 
most basic problem is to formulate $\mathrm{W}$ gravity without gauge fixing, and to couple it to some appropriate kind of matter from first principle.

\section{References}

[1] A.B.Zamolodchikov, Theor. Math. Phys. 65 (1985) 1205; V.A. Fateev and A.B.Zamolodchikov, Nucl. Phys. B280 [FS18] (1987) 644.

[2] A. Bilal and J.-L. Gervais, Phys. Lett. B206 (1988) 412; Nucl. Phys. B314 (1989) 646; Nucl. Phys. B318 (1989) 579.

[3] J.-L. Gervais, Phys. Lett. B160 (1985) 277; K. Yamagishi, Phys. Lett B205 (1988) 406; I. Bakas, Phys. Lett. B213 (1988) 313.

[4] M. Sato, RIMS Kokyuroku 439 (1981) 30; E. Date, M. Jimbo, M.Kashiwara and T.Miwa, "Transformation Groups for Soliton Equations" in Proc. of RIMS Symposium on Non-linear Integrable SystemsClassical Theory and Quantum Theory (Kyoto, Japan, May 1981) (World Scientific Publication Co. Singapore, 1983); G.Segal and G.Wilson, Pub. Math. IHES 61 (1985) 5.

[5] J.-L. Gervais, Y. Matsuo: Phys. Lett. 274B (1992) 309;

[6] J.-L. Gervais, Y. Matsuo: Comm. in Math. Phys. 152 (1993) 317.

[7] J.-L. Gervais, Y. Matsuo: Phys. Lett. 312B (1993) 285.

[8] A. Polyakov: Mod. Phys. Lett. A 2, 11 (1987) 893.

[9] J.-L. Gervais, Comm. in Math. Phys. 130, 257, (1990) .

[10] J.-L. Gervais, Phys. Lett. B243, 85, (1990) .

[11] E. Cremmer, J.-L. Gervais, Comm. in Math. Phys. 134, 619, (1990).

[12] O. Babelon, Phys. Lett. B215, (1988) 523.

[13] J.-L. Gervais, Comm. in Math. Phys. 138 138, 301, (1991) . 
[14] J.-L. Gervais, "Quantum group derivation of 2D gravity-matter coupling" Invited talk at the Stony Brook meeting String and Symmetry 1991, LPTENS preprint 91/22, Nucl. Phys. B391, 287, (1993).

[15] J.-L. Gervais, J. Schnittger, Phys. Lett. B315 , 258, (1993).

[16] Y. Matsuo: Phys. Lett. B227 (1989) 209.

[17] see, for instance, the related reviews in "String and symmetries 91", in Proceeding of the Stony Brook Meeting, World Scientific ed.

[18] E.Cremmer, J.-L. Gervais. J.-F. Roussel, "The genus-zero bootstrap of chiral vertex operators in Liouville theory", preprint LPTENS-93/29, Nucl. Phys. to be published.

[19] E.Cremmer, J.-L. Gervais. J.-F. Roussel, "The quantum group structure of 2D gravity and minimal models II: The genus zero chiral bootstrap" preprint LPTENS-93/02, hep-th 9302035, Comm. Math. Phys. to be published.

[20] J.-L. Gervais, J. Schnittger, "The many faces of the quantum Liouville exponentials" preprint LPTENS-93/30, hep-th 9308134, Nucl. Phys. to be published. 